\title{
Fuzzy Tier-based User Experience Prediction Scheme
}

\author{
Ahmed. A. A. Gad-EIRab \\ Department of Mathematics \\ Faculty of Science \\ Al-Azhar University-Cairo, Egypt
}

\author{
Kamal A. EIDahshan \\ Department of Mathematics \\ Faculty of science \\ Al-Azhar University-Cairo, Egypt
}

\author{
Mahmoud Embabi \\ Department of Mathematics \\ Faculty of Science \\ Al-Azhar University-Cairo, Egypt
}

\begin{abstract}
Building professional and efficient systems by using user experience became one of the important research activities that focus on the interactions between products, applications, designers, and users. Unfortunately, using user experience faces many problems. One of these problems is how to predict a user experience efficiently to build robust, effective, and flexible applications. To solve this problem, it is needed to design an optimal and efficient method for predicting user experience which includes behavior and emotions experiences. In this paper, a two-tier ranking scheme by using two multi-criteria decision making approaches is proposed. This proposed scheme considers a user experience as a sequence of executed actions or operations and it can predicate the most efficient user experience sequence of operations among a group of user experiences or experiences of individual users on a certain system or application. It uses the combination of two multi-criteria decision making approaches, the analytic hierarchy process (AHP) and the technique for order performance by similarity to ideal solution (TOPSIS) in Fuzzy environments to rank each operation or action in a user sequence. Based on operation rank, in the first tier, the proposed algorithm selects all sequential operations with the highest ranks. If there are sub goals are not satisfied in the first tier, then in the second tier, the algorithm ranks all unselected operations and add all operations with the highest ranks which satisfy these sub goals. This new scheme is presented as a flexible and efficient method for predicting user experience which will be help designers and developers in building professional systems and applications.
\end{abstract}

\section{Keywords}

Human computer interaction, User experience design, Fuzzy sets, AHP, TOPSIS.

\section{INTRODUCTION}

Designing a user experience (UX) became a critical issue for building professional and efficient systems due to the development of information technology schemes, HCI techniques and electronic devices. The user experience introduces new research activities that focused on the interactions between products, applications, designers, and users. Recently, a lot of industrial and technological companies have touched the importance of UX as a key success issue in product design [1]. The creating meaningful UX is not just usability but it goes far more. Therefore, it is essential to take into account other cognitive, socio-cognitive, and affective aspects of UX in the interaction process, such as users' enjoyment, brand loyalty, mental models, and aesthetic experience [2]. In addition, the user behavior is very important issue to be considered in designing UX.

In product design process, there are many interdependent designing attributes are considered as a consistent whole to create unique UX, especially to achieve valuable higher economic benefit and customer desires [3]. The evolution of user's emotional states and cognitive processes with choice decision making are the chain of human-product interactions [4]. Traditionally, most of designers concentrated on functional requirements for physical products and did not consider users' behavior and affective and cognitive needs. Recently, designers can utilize the new technologies, compose multimedia platforms with services, or use of sensory information for creating meaningful UX based on the context of work environments [5].

In decision making, human emotional experience plays a significant role towards product success [6]. Therefore, it is very important to consider the human dimension in design research [7]. Most of existing human decision making systems have been well addressed based on the user cognitive experiences. However, these systems miss the affective elements for modeling, analyzing and simulating human realization on UX in the predominant computational models [8]. Recent models based on behavioral decision theories focus on cognitive errors and heuristics in human decision making, but still ignore the role of emotion in human decision making $[9,10]$. Users' affective states often influence their experience at the time of decision making, so a single cognitive perspective is not optimal for analyzing human decisions for meaningful UX [10]. Recently, in [11] the integration of emotion and cognition has been driven by the intimate coupling of affective and cognitive decisions.

To meet user goals, in this paper, a two-tier ranking scheme based on fuzzy decision making approaches and category and activity theories is proposed. This proposed scheme considers a user experience as a sequence of executed actions or operations and it can predicate a most efficient user experience sequence of operations among a group of user experiences or experiences of individual users on a certain system or application. It uses the combination of two multicriteria decision making approaches, the analytic hierarchy process (AHP) and the technique for order performance by similarity to ideal solution (TOPSIS) in Fuzzy environments to rank each operation in a user sequence. Based on operation rank, in the first tier, the proposed algorithm selects the all sequential operations with the highest ranks. If there are sub goals are not satisfied in the first tier, then in the second tier, the algorithm ranks all unselected operations and add all operations with the highest ranks which satisfy these sub goals. This new scheme is presented as a flexible and efficient method for predicting user experience which will be help designers and developers in building professional systems and applications.

The rest of the paper is organized as the following. Section 2 includes a detailed survey of the related work. Section 3 introduces multi-criteria decision making approaches. Section 4 describes user experience prediction problem and its related assumptions. Section 5 describes a proposed user experience prediction scheme. Section 6 presents real scenario example and its analysis results. Finally, Section 7 concludes the paper. 


\section{RELATED WORK}

Most of user-centered design researchers were intensified affective perception of a product use and concentrated on a product functionality and usability aspects. However, they gave a slight concern of how affect influences behavior and emotion experiences of a user as a whole.

Several models were formulated for analyzing and predicting choice behavior and preference of a user in a variety of application contexts [12]. In [13], for designing a healthcare system a think-aloud protocol was applied to investigate cognitive requirements of nurses and physicians. To improve affective UX, there are a lot of research areas gave more attention for using a user affect an emotions such as users' imaginary expectation and momentary emotions in different contexts and at different points of time [14], [15]. In [16], the affective UX (AUX) was improved by selecting appropriate design elements that are able to extract positive emotions. Also, to deal with the uncertainty aspects, fuzzy set are integrated [17]. The quality function deployment, $Q F D$, is one of the most commonly used methods for representing user preferences in engineering design [18]. To map product features and functionality favored by users, a house of quality is formulated. For example, in [18] to design the B787 Dreamliner commercial aircraft the QFD was used to translate lifestyle, image, and psychological needs into design requirements. To understand the basic human needs for human experience design, there is increasing trend for studying of interaction between affect and cognition. For instance, Lisetti and Nasoz [19] examined that how affect interacts with cognition and developed a multimodal affective user interface for simulating human intelligence. An affective-cognitive decision framework was proposed for learning and decision making in [10]. In [20], the authors deducted that affect and cognition are highly interdependent because the phenomena themselves are coupled.

Most of existing works can build meaningful $\underline{\mathrm{UX}}$ model based on a user behavior or a user emotion separately and may not build a meaningful UX model based on both of them together. Also, none of them can represent a user behavior or a user emotion by using a unified model in UX design and they can not predict the optimal user experience design efficiently. So, a new scheme that considers a user experience as a sequence of executed actions or operations and it can predict the most efficient compound user operation sequence among experiences of a group of users.

\section{MULTI-CRITERIA DECISION MAKING APPROACHES}

\section{A. Fuzzy Sets and Fuzzy Number}

Zadeh (1965) introduced the Fuzzy Set Theory (FST) to deal with the uncertainty and ambiguous of data. A major contribution of FST is the capability of representing uncertain data. FST also allows mathematical operators and programming to be performed to the fuzzy domain. A Fuzzy Set (FS) is a class of objects with a continuum of grades of membership. Such a set is characterized by a membership function, which assigns to each object a grade of membership ranging "between" zero and one. To understand Fuzzy Set and Triangular fuzzy number in details, you can read their description in [22].

\section{B. Analytic Hierarchy Process (AHP)}

Bernoulli (1738) proposed the concept of utility function to reflect human persuit, such as maximum satisfaction, and von Neumann and Morgenstern (1947) presented the theory of game and economic behavior model, which expanded the studies on human economic behavior for multiple criteria decision making (MCDM) problems [16], an increasing amount of literature has been engaged in this field. The MCDM can be summarized in sex main steps as follows:

1) Define the nature of the problem.

2) Construct a hierarchy system for its evaluation Fig. 1

3) Select the appropriate evaluation model.

4) Obtain the relative weights and performance score of each attribute with respect to each alternative.

5) Determine the best alternative according to the synthetic utility values, which are the aggregation value of relative weights, and performance scores corresponding to alternatives.

6) Outrank the alternatives referring to their synthetic fuzzy utility values from Step 5.

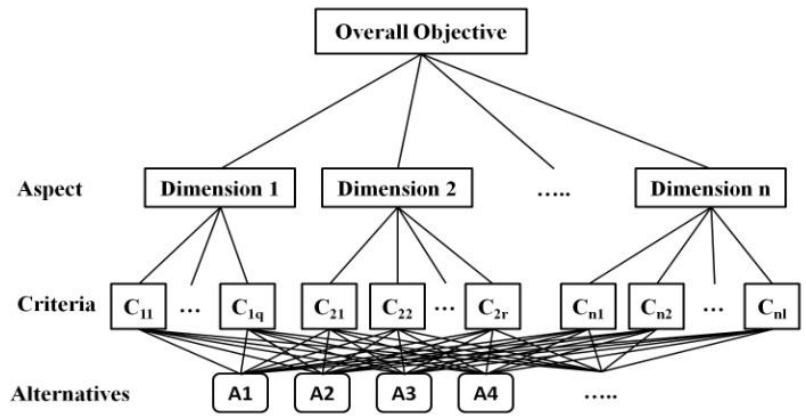

Fig. 1: Hierarchical system for MCDM

The analytic hierarchy process $(A H P)$ was proposed to derive the relative weights according to the appropriate hierarchical system. There are four methods, including the eigenvalue method, the geometric mean method, the linear programming method and the lambda-max method to derive the weights using the $A H P$. Only the eigenvalue method is employed to deal with crisp numbers and the other methods are adapted to handle the $A H P$ under fuzzy numbers [16]. To understand $A H P$, Fuzzy AHP, TOPSIS, Fuzzy TOPSIS methods in details, you can read their description in [22].

\section{USER EXPERIENCE PREDICTION PROBLEM}

In this section, the proposed definitions, assumptions, and models will be introduced then the user experience prediction (UXP) problem will be described.

\section{A. Definitions, Assumptions, and Models}

a user experience design process is defined as a quadruple system $Q(A T, A C, O P, C N)$ where, $A T=\left\{a v_{i}: 1 \leq i \leq M\right\}$ is a set of all activities in the system and $M$ is the total number of activities in the system, $A C=\left\{a c_{j}: 1 \leq j \leq N\right\}$ is a set of all actions in the system and $N$ is the total number of activities in the system, $O P=\left\{o p_{k}: 1 \leq k \leq K\right\}$ is a set of all operations in the system and $K$ is the total number of operations in the system, and $C N=\left\{c n_{l}: l \leq l \leq L\right\}$ and $L$ is the total number of logical conditions in the system. Also, each operation $o p_{k} \in O P$ consists of a set of sequential tasks which is denoted as $T S\left(o p_{k}\right)=\left\{t s_{r}: 1 \leq r \leq R\right\}$ where $R$ is the total number of tasks in operation $o p_{k}$. A set of user emotions is denoted as $E N=$ 
$\left\{e n_{s}: 1 \leq s \leq S\right\}$ where $S$ is the total number of detected user emotions.

In this paper, cCABM [21] model is used for describing user experience. There is a set of users $U$ use an application or system which is described by cCABM [21] model and each user $u \in U$ will use a limited sequence of operations to satisfy a certain activity goal in the system or application. Also, there are different sequences paths of operations to satisfy a certain activity goal. A sequence path which is executed by a user $u \in$ $U$ for activity $a c \in A C$ is denoted as $\operatorname{Seq}(u, a c)=$ $\left[o p_{1}, o p_{2}, \ldots, o p_{T}\right]$. A set of operations in $\operatorname{Seq}(u, a c)$ is denoted as $O P_{\text {Seq }(u, a c)}$. A set of action goals (short goals) of an activity $a v$ is denoted as $A C G(a v)=\left\{g_{j}: 1 \leq j \leq N\right\}$. Each action/operation goal (short goal) $g_{j}$ consists of a sub-short term goals and is denoted as sgoals $\left(u, o p_{i}\right)$, where number of these sub goals are $H_{i}$. The overall number of subgoals for this user activity is denoted as $Q$. There is a goal satisfaction percent for each operation $o p$ by user $u$ and is denoted by $\operatorname{OGSP}\left(u, o p_{i}\right)$ which means the ratio of number of satisfied sub goals, $h_{i}$, to the total number of sub goals, $H_{i}$, of an operation $o p_{i}$ and is defined as follows.

$$
\operatorname{OGSP}\left(u, o p_{i}\right)=\frac{h_{i}}{H_{i}}
$$

The total operation time which is taken by a sequence of operation to finish an operation is denoted as $\operatorname{OST}\left(u, o p_{i}\right)$. There is a maximum sequence time OST_MAX which is accepted by a user or a system for any user operation in the system. An operation time ratio is denoted as $\operatorname{OSTR}\left(u, o p_{i}\right)$ and is defined as follows.

$$
\operatorname{OSTR}\left(u, o p_{i}\right)=\frac{O S T\left(u, o p_{i}\right)}{O S T_{-} M A X}
$$

\section{B. UXP Problem Formulation}

Here, the UXP problem is how to predicate and construct the best combination sequence of operations for user experience efficiently which are selected from all operations that executed by users. The main difference between this description and the previous UXP description in FPUEA [22] is that FPUEA predicts and select the best operation sequence among the executed operation sequences of users while in this paper, the problem is how to predict and construct a new compound operation sequence by combining a set of operations which are selected based on their effectiveness.

The main objective of this model is achieving all of systems goals in a professional and helpful way such that this model must satisfy all related conditions of the system. Therefore, based on the assumptions and system models, the UXP problem can be described as follows.

Objective: Predict a best compound operation sequence,

$$
\operatorname{PreSeq}(U, a c)=\left\langle o p_{1}, o p_{2}, o p_{3}, \ldots, o p_{n}>\right.
$$

\section{Such that:}

$$
\begin{aligned}
& o p_{i} \in \bigcup_{\forall u \in U} \operatorname{Seq}(u, a c) \\
& G S P_{\text {PreSeq }(U, a c)} \geq \operatorname{Max}\{g \operatorname{sp}(u, a c), \forall u \in U\}
\end{aligned}
$$

$$
\operatorname{ASTR}_{\text {PreSeq }(U, a c)} \leq \operatorname{Max}\left\{S T R_{\text {Seq }(u, a c)}, \forall u U\right\}
$$

where $G S P_{\operatorname{PreSeq}(U, a c)}$ represents the goal satisfaction percent of predicted sequence, $g s p(u, a c)$ represents the goal satisfaction percent of a sequence which is executed by user $u$, $A S T R_{\text {PreSeq(U,ac) }}$ represents the total sequence time that will be taken by the predicated sequence, $S T R_{S e q(\mathrm{u}, a c)}$ represents the total sequence time that will be taken by the a sequence is executed by user $\mathrm{u}$, and $S T R \_M A X$ represents the maximum allowable time by the system for any user operation sequence.

Constraint (4) means that any selected operation $o p_{i}$ in $\operatorname{PreSeq}(U, a c)$ must belong to at least one a user sequence $\operatorname{Seq}(u, a c)$ for all $u$ in of $U$. Constraint (5) means that the satisfaction percent of the predicted sequence is larger than or equal to the maximum satisfaction percent among all users in $U$. Constraint (6) means that the time of the predicted sequence is less than or equal to the maximum time among all users in $U$.

\section{TWO-TIER USER EXPERIENCE PREDICTION SCHEME (TTUEPS)}

In this section, the proposed scheme for predicting user experience will be introduced. The proposed scheme called Two-Tier User Experience Prediction Scheme (TTUEPS). The architecture of predicting process of TTUEPS is shown in Fig. 2. As shown in Fig. 2, the input data for TTUPS is the set of operation sequences by all users and the output results is the predicted compound operation sequence which consists of many operations from different users (i.e., different colors means different users). TTUEPS constructs a hierarchal prediction mechanism consists of two tiers Main tier and Complement tier to obtain the final prediction operation sequence. Main tier composed of four steps which steps are: (1) Classification step: classifying all user operation sequences in levels and grouping all operation that belongs to the same level in a one set of operations (2) Calculation step: calculating the best weight for each operation in all sequences alternatives by using FAHP, (3) Evaluation step: evaluating all operations in all sequences alternatives by using FTOPSIS, and (4) Selection step: selecting the best operations among all operations in all sequence alternatives. At the end of these steps, the final produced operation sequence is considered as the best user experience predicted sequence for a system.

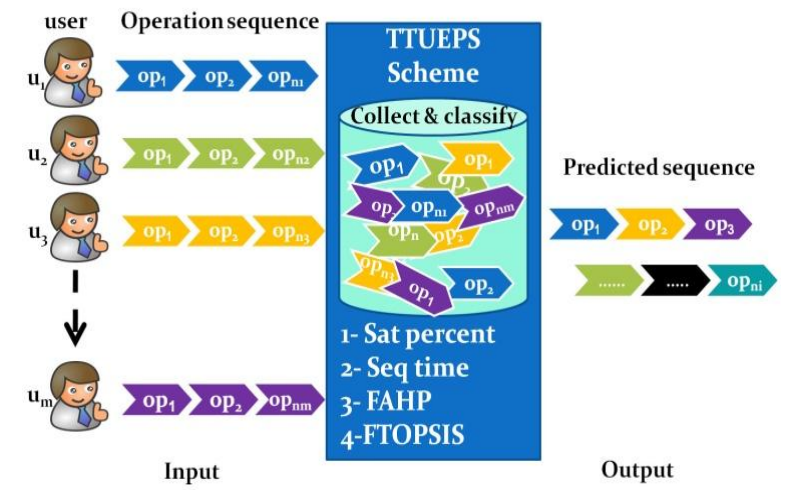

Fig. 2. Architecture of Predicting Process of TTUEPS

In case of existing some of unsatisfied subgoals with the predicted operation sequence that are achieved by some of operation sequences of users, TTUEPS executes the complement tier. This tier consists of five steps which are: (1) Classification step: classifying all remaining operations that contribute in achieving unsatisfied subgoals into dependent 
and independent sets (2) Calculation step: calculating the best weight for each operation in dependent and independent sets by using FAHP, (3) Evaluation step: evaluating all operation alternatives by using FTOPSIS, (4) Selection step: selecting the best operations among all operations, and (5) Insertion step: inserting each selected operation into the predicted operation sequence at its right place. In the rest of this section, the steps of each tier will be described in details.

\section{A. Main Tier of TTUEPS}

In this tier, TTUEPS constructs the main predicted operation sequence based on the ranking of operations that are constructing all available users operation sequences as follows.

\section{1) Main Classification step}

To rank each operation, firstly, TTUEPS classifies each user operation sequence into levels based on its execution sequence and its subgoals set. Secondly, it associates each level with a set of all operations that exist in this level among all user operation sequences which is denoted by $o_{l}$.

\section{2) Main calculation step}

In this step, for each operation level, TTUEPS uses FAHP to calculate the best weights for a goal satisfaction percent and user operation time for each operation in its associated set. So, firstly TTUEPS uses linguistic variables to describe goal satisfaction percent and user operation time. The defined linguistic variables are used to describe the estimated values for each user resulted operation and are shown in Table I and Table II for goal satisfaction percent and user operation time, respectively. By using FAHP process which is described in [22] and TTUEPS defined linguistic variables and their related ratio values, TTUEPS can get the best weighted values for user goal satisfaction percent and user operation time for each level.

\section{3) Main evaluation step}

In this step, TTUEPS uses FTOPSIS which is described in [22] and the resulted best weighted values which are resulted from step 2 (calculation step) to evaluate all operations that are used by different users in the same execution level .

Table I: Linguistic Values and their ratio values for a goal satisfaction of a user operation

\begin{tabular}{|l|l|}
\hline Linguistic values & Fuzzy numbers \\
\hline Very weak (VW) & $(0,0.01,0.5)$ \\
\hline Weak $(\mathrm{W})$ & $(0.01,0.5,0.6)$ \\
\hline Fair $(\mathrm{F})$ & $(0.5,0.6,0.75)$ \\
\hline Good $(\mathrm{G})$ & $(0.6,0.75,0.85)$ \\
\hline Very good (VG) & $(0.75,0.85,0.9)$ \\
\hline
\end{tabular}

Table III: the Set of goals and their subgoals of formatting scenario

\begin{tabular}{|c|c|}
\hline Goal & Subgoals \\
\hline \multirow{2}{*}{ A. Write Title Slide } & A1. Write Slide Show title. A2. Format the title \\
& $\begin{array}{r}\text { A3. Change Slide Design. } \quad \text { A4. Inset slide number and footer. } \\
\text { A5. Add animation. A6. shows slide design }\end{array}$ \\
\hline B. Write Outlines Slide & B1. Inserting new slide. B2. Write Slide Title \\
& B3. Format the title. B4. Write Outlines \\
& B5. Format the Outlines. B6. Add animation. \\
\hline
\end{tabular}

\begin{tabular}{|l|l|}
\hline Excellent (E) & $(0.85,0.9,1.0)$ \\
\hline Complete (C) & $(0.9,1.0,1.0)$ \\
\hline
\end{tabular}

Table II: Linguistic Values and their ratio values for user operation time

\begin{tabular}{|l|l|}
\hline Linguistic values & Fuzzy numbers \\
\hline Very short (VS) & $(0,0.01,0.5)$ \\
\hline Short (S) & $(0.01,0.5,0.7)$ \\
\hline Medium (M) & $(0.5,0.7,0.9)$ \\
\hline Long (L) & $(0.7,0.9,1.0)$ \\
\hline Very long (VL) & $(0.9,1.0,1.0)$ \\
\hline
\end{tabular}

In this paper, only two different criteria are considered: operation time delay and operation goal satisfaction. Note that, for operation time delay criterion, most of applications and systems needs a lower operation time delay while for operation goal satisfaction percent criterion, they needs a higher goal satisfaction percent. By using FTOPSIS process, TTUEPS can evaluate all user operations based on those two criteria. The resulted evaluation for operation time delay and operation goal satisfaction criteria are denoted as $\operatorname{ESTR}(u, o p$, $\left.o s_{l}\right)$ and $\operatorname{EGSR}\left(u, o p, o s_{l}\right)$, respectively.

\section{4) Main selection step}

In this step, TTUEPS will select or predict the best user operation among all ranked operations in the same execution level $B o p\left(o p, o s_{l}\right)$ by using the resulted evaluated values in step 2 (evaluation step). To predict the best operation, TTUEPS will use accumulated performance for each user operation which is defined as follows.

$$
\begin{aligned}
& \operatorname{ACP} \operatorname{erf}\left(u, o p, o s_{l}\right)= \\
& \quad w_{1} x \frac{1}{\operatorname{ESTR}\left(u, o p, o s_{l}\right)}+w_{2} x \operatorname{EGSR}\left(u, o p, o s_{l}\right)
\end{aligned}
$$

where $w_{1}$ represents weight of sequence time ratio and $w_{2}$ represents weight of goal satisfaction ratio. The application will commit to determine the values of $w_{1}$ and $w_{2}$ such that:

$$
w_{1}+w_{2}=1
$$

Based on this accumulated performance, TTUEPS calculates it for each user operation and then sorts all values in descending order. Finally, TTUEPS selects the operation with highest value as the best predicted operation for this execution level. Finally, TTUEPS constructs the predicated operation sequence of an action after finishing all steps for each level in action execution sequence. The resulted predicated sequence is denoted as MainPred $(U, a c)$ where its set of selected operations is $O P_{\text {MainPred }(U, a c)}$. Also, the unsatisfied subgoals in this tier is denoted as UnsatGoals $(U, a c)$. 


\begin{tabular}{|c|c|}
\hline & B7. Typing agenda. \\
\hline C. Write First Slide & $\begin{array}{l}\text { C1. Inserting new slide. C2. Write slide title } \\
\text { C3. Format the title. C4. Write slide contents } \\
\text { C5. Format the paragraph. C6. Add animation }\end{array}$ \\
\hline D. Write second slide & $\begin{array}{l}\text { D1. Inserting new slide. D2. Write slide title } \\
\text { D3. Format the title. D4. Write slide contents } \\
\text { D5. Format the paragraph. D6. Add animation }\end{array}$ \\
\hline E. Write third slide & $\begin{array}{l}\text { E1. Inserting new slide. E2. Write slide title } \\
\text { E3. Format the title. } \quad \text { E4. Write slide contents } \\
\text { E5. Format the paragraph. E6. Write Sub title (Functions) } \\
\text { E7. Insert list of Functions. } \quad \text { E8. Write list of Functions. } \\
\text { E9. Add animation }\end{array}$ \\
\hline F. Write fourth slide & $\begin{array}{l}\text { F1. Inserting new slide. F2. Write slide title } \\
\text { F3. Format the title. } \quad \text { F4. Write slide contents } \\
\text { F5. Format the paragraph. F6. Add animation }\end{array}$ \\
\hline G. Write fifth slide & $\begin{array}{l}\text { G1. Inserting new slide. G2. Write slide title } \\
\text { G3. Format the title. G4. Write slide contents } \\
\text { G5. Format the paragraph. G6. Add animation }\end{array}$ \\
\hline H. Adding presentation style & $\begin{array}{l}\text { H1. Adding Slides transitions. H2. Adding slides layout } \\
\text { H3: adjusting slide resolution }\end{array}$ \\
\hline
\end{tabular}

\section{B. Complement Tier of TTUEPS}

In case of existing some of unsatisfied subgoals with the predicted operation sequence that are achieved by some of operation sequences of users, TTUEPS executes the complement Tier in five steps: Complement Classification step, Complement Calculation step, Complement Evaluation step, Complement Selection step, and Complement Insertion step. The complement calculation, evaluation, selection steps are the same as the main calculation, evaluation, selection steps in the main tier but they will be applied on dependent and independent sets which are produced from complement classification step, Therefore, in this complement tier, the only complement classification and insertion steps will be described.

\section{1) Complement classification step}

In this step, TTUEPS, collects all unselected operations, $\operatorname{UnSelOp}(U, a c)$, that contribute in achieving unsatisfied subgoals such that these operations do not have any common satisfied subgoal with the selected operations in the main predicated sequence MainPred $(U, a c)$.This is because, if there is a common satisfied subgoal among them, the same operation steps that executed by different users will be appeared many times in the final operation sequence. This condition is defined as follows.

$\operatorname{sgoals}\left(u, o p_{i}\right) \cap \operatorname{sgoals}\left(u, o p_{j}\right)=\phi, \forall u \in U$,

$o p_{i} \in U n \operatorname{SelOp}(U, a c), o p_{j} \in O P_{\operatorname{Main} \mathrm{Pred}(U, a c)}$

After constructing the unselected operations set, UnSelOp $(U, a c)$, TTUEPS classifies these collected operations into two groups: (a) Dependent group: which contains any operation that depends on one or more other operations in
$O P_{\text {MainPred(U,ac). In other words, this operation must come after }}$ or/and before specific operations in OP $P_{\text {MainPred }(U, a c)}$. (b) Independent group: which contains any operation that does not depend on any operation in $O P_{\operatorname{MainPred}(U, a c)}$. In other words, this operation can be set in between any two operations in the resulted predicted sequence of main tier MainPred $(U, a c)$. TTUEPS uses the input and output parameters of each operation to define the dependency between all operations. By using this relationship, it can classify all unselected operations into dependent and independent groups. The resulted dependent and independent groups are denoted as $\operatorname{Dep}(U, a c)$ and $\operatorname{Indep}(U, a c)$, respectively.

\section{2) Calculation, evaluation, and selection steps}

After this classification, TTUEPS executes the complement calculation, evaluation, and selection steps to get the selected operations for inserting them in the resulted predicted sequence of main tier MainPred $(U, a c)$. The selected dependent and independent sets are denoted as $S \_D e p(U, a c)$ and $S \_$Indep $(U, a c)$, respectively. Finally, TTUEPS executes the insertion step which will be described in the next subsection.

\section{3) Complement insertion step}

In this step, TTUEPS inserts each operation $S \_\operatorname{Dep}(U, a c)$ and $S \_I n d e p(U, a c)$ in its right place with MainPred $(U, a c)$ predicted sequence. Firstly, for any operation in $S \_D e p(U, a c)$, TTUEPS finds it right place by searching for its related operations in $O P_{\text {MainPred }(U, a c)}$. Secondly, for any operation in $S \_I n d e p(U, a c)$, TTUEPS inserts it in any place between two independent operations with MainPred $(U, a c)$. At the end of this insertion step, TTUEPS constructs the final predicated operation sequence, $\operatorname{PreSeq}(U, a c)$. 
Table 4. Executed operations, satisfied subgoals and time

\begin{tabular}{|c|c|c|c|}
\hline $\begin{array}{l}\text { User } \\
\text { ID }\end{array}$ & Operation & Satisfied subgoals & $\begin{array}{c}\text { Time } \\
\text { (seconds) }\end{array}$ \\
\hline \multirow{8}{*}{ U1 } & Op1 & $\mathrm{A} 1, \mathrm{~A} 2, \mathrm{~A} 3, \mathrm{~A} 5, \mathrm{~A} 6$ & 190 \\
\hline & Op2 & B3, B4, B5, B6, B7 & 155 \\
\hline & Op3 & $\mathrm{C} 1, \mathrm{C} 2, \mathrm{C} 3, \mathrm{C} 5$ & 110 \\
\hline & Op4 & $\mathrm{D} 1, \mathrm{D} 2, \mathrm{D} 3, \mathrm{D} 4, \mathrm{D} 5$ & 60 \\
\hline & Op5 & E1, E3, E4, E6,E7, E8 & 300 \\
\hline & Op6 & F1, F2, F3, F6 & 165 \\
\hline & Op7 & G1, G2, G4 & 170 \\
\hline & Op8 & $\mathrm{H} 1, \mathrm{H} 2$ & 60 \\
\hline \multirow{7}{*}{$\mathrm{U} 2$} & Op1 & A1, A2, A5 & 30 \\
\hline & Op2 & $\mathrm{B} 1, \mathrm{~B} 2, \mathrm{~B} 8$ & 120 \\
\hline & Op3 & $\mathrm{C} 1, \mathrm{C} 2, \mathrm{C} 4, \mathrm{C} 6$ & 180 \\
\hline & Op4 & D1, D2, D3, D5, D6 & 180 \\
\hline & Op5 & E1, E2, E4, E6, E9 & 180 \\
\hline & Op6 & $\mathrm{F} 1, \mathrm{~F} 2, \mathrm{~F} 3, \mathrm{~F} 6$ & 120 \\
\hline & Op7 & G2, G3, G5, G6 & 120 \\
\hline \multirow{3}{*}{ U3 } & Op1 & $\mathrm{A} 1, \mathrm{~A} 2, \mathrm{~A} 5$ & 169 \\
\hline & Op2 & B1, B2, B4, B5, B6 & 158 \\
\hline & Op3 & $\mathrm{C} 1, \mathrm{C} 2, \mathrm{C} 3, \mathrm{C} 5, \mathrm{C} 6$ & 163 \\
\hline \multirow{8}{*}{ U4 } & Op1 & $\overline{\mathrm{A} 1, \mathrm{~A} 2, \mathrm{~A} 4, \mathrm{~A} 5}$ & 150 \\
\hline & Op2 & $\mathrm{B} 1, \mathrm{~B} 2, \mathrm{~B} 4, \mathrm{~B} 7$ & 140 \\
\hline & Op3 & $\mathrm{C} 1, \mathrm{C} 2, \mathrm{C} 3, \mathrm{C} 5$ & 70 \\
\hline & Op4 & D1, D2, D3, D4, D5 & 60 \\
\hline & Op5 & E1, E2, E6, E7, E8 & 240 \\
\hline & Op6 & $\mathrm{F} 1, \mathrm{~F} 4, \mathrm{~F} 5$ & 90 \\
\hline & Op7 & G1, G4 & 120 \\
\hline & Op8 & $\mathrm{H} 1, \mathrm{H} 2$ & 60 \\
\hline
\end{tabular}

\section{REAL SCENARIO EXAMPLE AND DISCUSSION}

In this section, firstly, the real scenario example which is experimented in the research lab to evaluate the proposed TTUEPS is introduced. Then its results will be discussed.

\section{A. A real Scenario Description}

In the proposed real scenario, the user activity is a presentation formatting which is a type of word and animated processing activities. A set of users were asked to do this presentation formatting activity in the research lab by using a PC and Laptop machines (in this evaluation, the different specifications among machines are ignored). The number of cooperated users was 4 users. The tested presentation consists of a set of paragraphs and figures with a specific title. A set of goals and their subgoals were set for this formatting action scenario as shown in Table III. The number of formatting action goals is 8 goals and each goal has a set of subgoals as shown in Table III. As shown in Table III, the overall number of subgoals, $Q$, in this scenario was 50. Finally, each user was asked to format the tested presentation and record the used operation sequence and the time and the set of subgoals for each operation.
Table 5: Levels of all operations in the formatting scenario

\begin{tabular}{|l|l|}
\hline Level & The set of operations in each level \\
\hline 1 & Op1(U1), Op1(U2), Op1(U3), Op1(U4) \\
\hline 2 & Op2(U1), Op2(U2), Op2(U3), Op2(U4) \\
\hline 3 & Op3(U1), Op3(U2), Op3(U3), Op3(U4) \\
\hline 4 & Op4(U1), Op4(U2), Op4(U4) \\
\hline 5 & Op5(U1), Op5(U2), Op5(U4) \\
\hline 6 & Op6(U1), Op6(U2), Op6(U4) \\
\hline 7 & Op7(U1), Op7(U2), Op7(U4) \\
\hline 8 & Op8(U1), Op8(U4) \\
\hline
\end{tabular}

\section{B. A real Scenario Results and Discussion}

The results of the formatting scenario are collected from all users. Table IV shows all operations which were executed by each user with their satisfied subgoals and their times to format the tested presentation and to satisfy his goals and their subgoals. The proposed TTUEPS will be evaluated by using these results as follows.

\section{B.1. Results of TTUEPS Main tier}

1) Classification step results: as described in the classification step of the TTUEPS main tier, Table V shows all levels of the operation sequence for each user and the set of all operations exist in each level based on its execution sequence and its subgoals. As shown in Table V, there are eight levels and each level has a different number of operations.

Table VI: $g_{s p}(u, a c)$ and $S T R_{S e q(\mathrm{u}, a c)}$ for each user sequence, $\operatorname{OGSP}(u, o p)$ and $\operatorname{OSTR}(u, o p)$ of each operation

\begin{tabular}{|c|c|c|c|c|c|}
\hline $\begin{array}{l}\text { User } \\
\text { ID }\end{array}$ & Op & $\begin{array}{l}\text { OGSP } \\
(u, o p)\end{array}$ & $\begin{array}{l}\text { OSTR } \\
(u, o p)\end{array}$ & $g s p(u, a c)$ & $\begin{array}{l}S T R_{S e q(\mathrm{u}, a c)} \\
\text { (minutes) }\end{array}$ \\
\hline \multirow{8}{*}{ U1 } & Op1 & 0.833 & 0.63 & \multirow{8}{*}{0.68} & \multirow{8}{*}{20.17} \\
\hline & Op2 & 0.625 & 0.516 & & \\
\hline & Op3 & 0.667 & 0.367 & & \\
\hline & Op4 & 0.833 & 0.2 & & \\
\hline & Op5 & 0.667 & 1.0 & & \\
\hline & Op6 & 0.667 & 0.55 & & \\
\hline & Op7 & 0.5 & 0.567 & & \\
\hline & Op8 & 0.667 & 0.2 & & \\
\hline \multirow{7}{*}{$\mathrm{U} 2$} & Op1 & 0.5 & 0.1 & \multirow{7}{*}{0.62} & \multirow{7}{*}{15.5} \\
\hline & Op2 & 0.375 & 0.4 & & \\
\hline & Op3 & 0.667 & 0.6 & & \\
\hline & Op4 & 0.833 & 0.6 & & \\
\hline & Op5 & 0.556 & 0.6 & & \\
\hline & Op6 & 0.667 & 0.4 & & \\
\hline & Op7 & 0.667 & 0.4 & & \\
\hline \multirow{3}{*}{ U3 } & Op1 & $\begin{array}{l}0.5 \\
\end{array}$ & 0.563 & \multirow{3}{*}{0.26} & \multirow{3}{*}{8.17} \\
\hline & Op2 & 0.625 & 0.527 & & \\
\hline & Op3 & 0.833 & 0.543 & & \\
\hline \multirow{8}{*}{ U4 } & Op1 & 0.667 & 0.5 & \multirow{8}{*}{0.58} & \multirow{8}{*}{15.5} \\
\hline & Op2 & 0.5 & 0.467 & & \\
\hline & Op3 & 0.667 & 0.233 & & \\
\hline & Op4 & 0.833 & 0.2 & & \\
\hline & Op5 & 0.556 & 0.8 & & \\
\hline & Op6 & 0.5 & 0.3 & & \\
\hline & Op7 & 0.333 & 0.4 & & \\
\hline & Op8 & 0.667 & 0.2 & & \\
\hline
\end{tabular}


Table 7: The linguistic values and evaluated results of Main tier by FAHP and FTOPSIS

\begin{tabular}{|c|c|c|c|c|c|c|}
\hline $\begin{array}{l}\text { User } \\
\text { ID }\end{array}$ & Op & $\begin{array}{l}\text { Linguistic } \\
\text { Satisfaction percent }\end{array}$ & $\begin{array}{l}\text { Linguistic } \\
\text { operation time }\end{array}$ & $\begin{array}{l}\text { Evaluated } \\
\text { Satisfaction percent }\end{array}$ & $\begin{array}{l}\text { Evaluated } \\
\text { operation time }\end{array}$ & $\begin{array}{l}\text { Final evaluation } \\
\text { ACPref(u,op, os })\end{array}$ \\
\hline \multirow{8}{*}{ U1 } & Op1 & Very good & Medium & 0.8 & 0.589 & 1.249 \\
\hline & Op2 & Good & Medium & 0.571 & 0.462 & 1.368 \\
\hline & Op3 & Good & Short & 0.6 & 0.297 & 1.984 \\
\hline & Op4 & Very good & Very short & 0.8 & 0.111 & 4.905 \\
\hline & Op5 & Good & Very long & 0.625 & 1.0 & 0.813 \\
\hline & Op6 & Good & Medium & 0.6 & 0.5 & 1.3 \\
\hline & Op7 & Fair & Medium & 0.4 & 0.529 & 1.145 \\
\hline & Op8 & Complete & Very short & 0.501 & 0.111 & 4.755 \\
\hline \multirow{7}{*}{$\mathrm{U} 2$} & Op1 & Fair & Very short & 0.4 & 0.0 & 1.2 \\
\hline & Op2 & Weak & Short & 0.286 & 0.333 & 1.645 \\
\hline & Op3 & Good & Medium & 0.6 & 0.556 & 1.199 \\
\hline & Op4 & Very good & Medium & 0.8 & 0.556 & 1.299 \\
\hline & Op5 & Fair & Medium & 0.501 & 0.556 & 1.15 \\
\hline & Op6 & Good & Short & 0.6 & 0.333 & 1.802 \\
\hline & Op7 & Good & Short & 0.6 & 0.333 & 1.802 \\
\hline \multirow{3}{*}{ U3 } & Op1 & Fair & Medium & 0.4 & 0.514 & 1.173 \\
\hline & Op2 & Good & Medium & 0.571 & 0.474 & 1.34 \\
\hline & Op3 & Very good & Medium & 0.8 & 0.492 & 1.416 \\
\hline \multirow{8}{*}{$\mathrm{U} 4$} & Op1 & Good & Medium & 0.6 & 0.444 & 1.426 \\
\hline & Op2 & Fair & Short & 0.429 & 0.408 & 1.44 \\
\hline & Op3 & Good & Very short & 0.6 & 0.148 & 3.678 \\
\hline & Op4 & Very good & Very short & 0.8 & 0.111 & 4.905 \\
\hline & Op5 & Fair & Long & 0.501 & 0.778 & 0.893 \\
\hline & Op6 & Fair & Short & 0.4 & 0.772 & 0.848 \\
\hline & Op7 & Weak & Short & 0.199 & 0.333 & 1.601 \\
\hline & Op8 & Complete & Very short & 0.501 & 0.111 & 4.755 \\
\hline
\end{tabular}

2) Calculation step results: By using calculation step of TTUEPS main tier, TTUEPS can get the best weighted values for user subgoals satisfaction percent, $\operatorname{OGSP}(u, o p)$, and user operation time, $\operatorname{OSTR}(u, o p)$, for each level by using FAHP. The result of this calculation step is shown in Table VI. Where the $O S T \_M A X$ value was 300 seconds.

3) Evaluation step results: By using evaluation step of TTUEPS main tier, TTUEPS can evaluate all user operations in each level. The corresponding linguistic values of fuzzy evaluated results are shown in Table VI. Then, by using FTOPSIS, $\operatorname{OGSP}(u, o p)$ and $\operatorname{OSTR}(u, o p)$ of each operation are evaluated as shown in Table VI. Where the desired case and the worst case of satisfaction percent are 1.0 (total number of subgoals of this level) and 1/H (one subgoal), respectively. Also, the desired case and the worst case of operation time ration are 0.1 (30 seconds) and 1.0 (300 seconds), respectively. Also, the values of $w_{1}$ and $w_{2}$ were 0.5 .

4) Selection step results: By using selection step of TTUEPS main tier, TTUEPS selects the best user operation among all ranked operations in the same execution level $B o p\left(o p, o_{s l}\right)$ by using the resulted evaluated values in its evaluation step. These selected operations are highlighted in Table VII by gray color. As a result, the main prediction sequence, $\operatorname{MainPred}(U, a c)$ as follows.

MainPred $(U, a c)=\langle O p 1(U 4), O p 2(U 2), O p 3(U 4), O p 4(U 1)$, $O p 5(U 2), O p 6(U 2), O p 7(U 2), O p 8(U 1)>$

Based on this predicated sequence, the set of unsatisfied subgoals UnsatGoals $(U, a c)$ is $\{\mathrm{A} 3, \mathrm{~A} 6, \mathrm{~B} 3, \mathrm{~B} 4, \mathrm{~B} 5, \mathrm{~B} 6, \mathrm{~B} 7$,
C4, C6, D6, E3, E7, E8, F4, F5, G1, G4, H3 \}. So, TTUEPS executes its complement tier to include these subgoals in the final predicted sequence.

\section{B.2. Results of TTUEPS Complement tier}

1) Classification step results: as described in the classification step of the TTUEPS complement tier, the unselected operations $\operatorname{UnSelOp}(U, a c)$ is $\{O p 2(U 1)$, $O p 7(U 4)\}$. By using, the input and output parameters of each operation, the $\operatorname{Dep} G(U, a c)$ and $\operatorname{Indep} G(U, a c)$ sets are $\{O p 7(U 4)\}$, and $\{O p 2(U 1)\}$, respectively. Where $O p 7(U 4)$ depends on $O p 7(U 2)$ as input.

2) Calculation, evaluation, and selection steps results: As described in the complement tier of TTUEPS, the $S \_D e p G(U, a c)$ and $S \_I n d e p G(U, a c)$ sets are $\{O p 7(U 4)\}$, and $\{O p 2(U 1)\}$, respectively. These selected operations are highlighted in Table VII by dark gray color.

3) Insertion step results: By using insertion step of TTUEPS complement tier, TTUEPS will insert each selected operation that belongs to $S \_D e p G(U, a c)$ or $S \_I n d e p G(U, a c)$ in its right position in MainPred $(U, a c)$ to complete the predicted operation sequence and get the final predicted sequence, $\operatorname{PreSeq}(U, a c)$, for this formatting activity. As a result, the main prediction sequence, $\operatorname{PreSeq}(U, a c)$, as follows.

$\operatorname{PreSeq}(U, a c)=\langle O p 1(U 4), \underline{O p 2(U 1)}, \quad O p 2(U 2), \quad O p 3(U 4)$, Op4(U1), Op5(U2), Op6(U2), Op7(U2), Op7(U4),Op8(U1)>

As a result, the set of final satisfied subgoals are $\{\mathrm{A} 1, \mathrm{~A} 2, \mathrm{~A} 4$, A5, B1, B2, B3, B4, B5, B6, B7, B8, C1, C2, C3, C5, D1, D2, 
D3, D4, D5, E1, E2, E4, E6, E9, F1, F2, F3, F6, G1, G2, G3, G4, G5, G6, H1, H2 \}. So, the total number of subgoals by using this final predicted sequence is 38 and satisfaction ratio is 0.76 (i.e., 38/Q) which is larger than 0.68 (this is the maximum satisfaction percent among all users which was satisfied by using the operation sequence of $\mathbf{U} \mathbf{1}$ as shown in Table VI). As Also, the total time of this final sequence is 1155 seconds (19.25 minutes) which is less than 20.17 (this is the maximum sequence time among all users which was taken by using the operation sequence of $\mathbf{U} \mathbf{1}$ as shown in Table VI). As shown in this final prediction sequence, to do a certain user activity, TTUEPS can predict the best operation sequence that maximizes the number of satisfied subgoals and minimizes the total time as much as possible. As a result, this sequence for predicting user experience can help designers and developers for building professional systems and applications.

\section{CONCLUSION}

In this paper, a two tier ranking predicting algorithm for predicting user experience called Two-Tier User Experience Prediction Scheme (TTUEPS) was proposed. TTUEPS consists of two tiers: Main tier and complement tier. The proposed algorithm considers a user experience as a sequence of executed actions or operations and it can construct a most efficient user experience predicated compound sequence among experiences of many users or experiences of individual users on a certain system or application based on the combination of two multi-criteria decision making approaches, FAHP and FTOPSIS, to rank each operation in a user sequence. Based on operation rank, in the main tier, the proposed algorithm selects the all sequential operations with the highest ranks. If there are sub goals are not satisfied in the first tier, then in the complement tier, the algorithm ranks all unselected operations and add all operations with the highest ranks which satisfy these subgoals. The proposed algorithm can predict the most efficient compound sequence of operations for doing a certain activity using an application or system software. Also, this paper introduced a real scenario example to evaluate TTUEPS. This case example validates that TTUEPS can be used as an efficient and helpful tool for predicting a user experience which can be used by researchers, developers, and designers for building a lot of professional, smart, and interactive applications.

\section{REFERENCES}

[1] Nielsen , Z. J. 2008. Usability ROI Declining, But Still Strong, in Nielsen Norman research group, Jan. 22.

[2] Law, E. L. C. and van Schaik, P. 2010. Modelling user experience, at an agenda for research and practice," Interact. Comput., 22(5), 313-322,

[3] Gould , D. 2010. Product ecosystems and service design, Available: http://www.psfk.com/2010/01.

[4] Picard, R.W. 1997. Affective Computing, in the MIT Press, Cambridge, Massachusetts.

[5] Hekkert, P. and Schifferstein, H. N. J. 2008. Introducing product experience, in Product Experience, P. Hekkert and H. N. J. Schifferstein, Eds. New York: Elsevier, 1-8.

[6] Brown, A.S. 2008. The New Point of View: Focus on Design for Human Factors, ASME Mechanical Engineering Magazine.
[7] Papalambros, P.Y. 2010. The human dimension, in ASME Journal of Mechanical Engineering, 132(5).

[8] Ahn, H. 2010. Modeling and analysis of affective influences on human experience, prediction, decision making, and behavior, MIT, thesis, America.

[9] BrandstÃdtter, E., Gigerenzer, G., and Hertwig, R. 2006. The priority heuristic: Making choices without trade-offs, Psychological Review, 113(2), 409-432,

[10] Ahn, H. and Picard, R.W. 2005. Affective-cognitive learning and decision making: a motivational reward framework for affective agents, in the $1^{\text {st }}$ International Conference on Affective Computing and Intelligent Interaction (ACII 2005), Beijing, China.

[11] Scherer, K.R., Shorr, A., and Johnstone, T. 2001 Appraisal processes in emotion: theory, methods, research, in Oxford University Press, Canary, NC.

[12] Zhou, F. and Jiao, R. J. 2013. An improved user experience model with cumulative prospect theory, in Procedia Computer Science, 16, 870-S877.

[13] Johnson, C. M. and Turley, J. P. 2006. The significance of cognitive modeling in building healthcare interfaces, Int. J. Med. Inf., 75(2), 163-172.

[14] Hassenzahl, M., Diefenbach, S., and Goritz, A. 2010. Needs, affect, and inter- active products, and facets of user experience, Interact. Comput., 22(5), 353-362.

[15] Jiao, J., Zhang, Y., and Helander, M. G. 2006. A Kansei mining system for affective design, in Expert Syst. Appl., $30(4), 658-673$

[16] Griffin, A. and Hauser, J.R. 1993. The voice of the customer, Marketing Science, 12, 1-27.

[17] Scott, M. and Antonsson, E.K. 1998. Aggregation functions for engineering design tradeoffs, Fuzzy Sets and Systems, 99(3), 253-264.

[18] Mazur, G.H. 2005. Life QFD: Incorporating emotional appeal in product development, in 17th Symposium on Quality Function Deployment, Portland.

[19] Lisetti, C.L. and Nasoz, F. 2002. MAUI: A multimodal affective user interface, in: The tenth ACM international conference on Multimedia, Juan-les- Pins, France, 161170 .

[20] Storbeck, J. and Clore, G.L. 2007. On the interdependence of cognition and emotion, in Cognition and Emotion, 21(6), 1212 -1237.

[21] Ahmed A. A. G., ElDahshan, K. A., and Embabi M. E. 2014. Conditional Activity-Based Model for Describing User Experience Using Category Theory, in International Journal of Computer Trends and Technology (IJCTT), 18(6).

[22] Ahmed A. A. G., ElDahshan, K. A., and Embabi M. E. 2015. Fuzzy Predictable Algorithm for User Experience, in International Journal of Computer Trends and Technology (IJCTT), 20(1), 50-58. 